\title{
THE RELATIONSHIP OF DENTISTRY TO OPHTHALMOLOGY
}

\author{
By ROBERT BLUE, A.B., M.D., Chicago, Illinois
}

$\mathrm{O}$ PHTHALMOLOGY and dentistry are similarly regarded by a vast number of people. The treatment of diseases of both the eyes and the teeth is too often regarded as disassociated with the treatment of pathology in other parts of the body. The fact that the eyes and the teeth are but highly specialized organs of the body, participating in many of its diseases and reflexly influencing the functioning of the entire body, is lost to view. While this fact may be known as an abstract idea to many occulists and dentists proper emphasis is not given to it.

The most striking example of the close association of the eyes and the teeth in a pathological syndrome is in that classical triad of symptoms known as Hutchinson's triad, which is pathognomonic of hereditary syphilis. Strangely enough the findings of this triad are distributed among three highly specialized departments of medicine, viz.: ophthalmology, otology, and dentistry. Almost invariably with the interstitial keratitis, the deafness, and the typically malformed teeth of the second dentition constituting this triad will be found other changes indicative of hereditary syphilis. These changes we will not dwell upon here, but it might be worth while to note that the typically pegged and notched teeth of the second dentition are not the only changes wrought in the teeth by this disease. Nor- mally the molar teeth are inverted truncated cones, i.e., they are narrower across their necks than across their crownscauliflower-shaped, as it were. They are truncated cones with the cutting surfaces corresponding to the bases of the cones. Attention has been called to the fact that in hereditary syphilis the molar teeth are truncated cones with the bases of the cones at the gingival margin, so that in this case they are narrower across the crowns than at the necks.

Less universally recognized is the association of diseases of the teeth and of the crystalline lens. At first thought this might appear to be a far-fetched theory, but when it is remembered that embryologically the teeth and the crystalline lens are both ectodermal in origin, it no longer seems strange that some points of contact should remain unimpaired thru the various processes of differentiation. When this fact is taken into consideration it seems no longer strange to find a profound constitutional disturbance producing pathology in the teeth and the crystalline lens. Rickets furnishes an example of this association.

The changes wrought in the teeth by rickets are characteristics and often profound. In the milder cases the enamel is furrowed horizontally. The incisors show this horizontal furrowing most plainly, tho it may be marked on all the teeth. Carried to greater lengths 
these changes result in the familiar terraced teeth of rickets. In the still more severe cases the teeth are aborted and the enamel is wanting in part or even in whole, the latter condition obtaining only in the most severe and neglected cases.

There is a type of cataract found in the eyes of children to which the names lamellar and perinuclear have been applied. This is the most frequent type of cataract found in children. Its association with rickets is sufficiently frequent to have given rise to the theory that it is the result of rickets. We know from clinical experience that in children we frequently find rachitic teeth associated with lamellar cataract, and we also find both embryological and theoretical reasons why the teeth and the eyes should be thus drawn together in a clinical picture.

In the cases sighted the pathology in the teeth and the eyes is a manifestation in these organs of a common general disease (provided, of course, that in the latter case the theory of Horner as to the cause of lamellar cataract is accepted). There are, on the other hand, frequent illustrations of diseases of the teeth being the direct cause of disease in the eyes or their appendages.

In this connection I need but call your attention to those cases of orbital cellulitis, where the retrobulbar phlegmon is a direct extension from a suppurative periostitis of the alveolar process of the superior maxilla. In these cases the inflammation in the orbital tissues occurs as a direct extension from periostitis of the teeth or appears after the extraction of diseased teeth. I do not want to convey the idea that dental disease is a frequent cause of orbital cellulitis by direct extension, but that dental disease must be included in the etiological causes of orbital cellulitis is beyond question.

More obscure perhaps from the standpoint of diagnosis than any of the con- ditions mentioned above are those cases of painful and irritable eyes which are due to reflex causes. I refer particularly to those eye symptoms which find their primary cause in stimuli applied to the terminations of the fifth nerve in and about the dental mechanism. The fifth nerve is not an intelligent localizing nerve at all times. Stimulation of the terminations in one branch is likely to be interpreted in terms of pain to some other point than that to which the stimulus is applied. The pain may be referred to some other branch or division of the nerve. Thus in the headache of eye strain the stimulation to the branches of the fifth nerve located in the eyeball, particularly in the ciliary body, is referred to the meningeal branches.

In a similar manner stimulation of the terminations of the fifth nerve in the upper jaw may be interpreted in terms of symptoms in the eyes. There is, of course, no reason to assume that the stimuiation must be applied to the terminations of the fifth nerve in the upper jaw rather than elsewhere in the distribution of this nerve. I mention it in this manner for the sake of emphasis only and for the further reason that in the most striking examples of eye symptoms due to dental irritation which have come under my observation the point to which the stimulation has been applied has been in the upper jaw. An illustrative case may serve to still further emphasize this point.

The case is that of a young man wh.n was troubled by recurrent attacks of pain, lacrymation and redness of the left eye. Cold, wind, or an unusual amount of use would initiate an attack, according to his history. So persistent and annoying were the attacks that he discontinued his college career on account of them. I placed him in the hospital and thoro physical examination by a competent internist re- 
vealed nothing except a crowned and apically infected upper incisor. Proper treatment of the dental condition cut short the attacks.

In this group of cases we must include those cases of unerupted teeth which by their irritation to the terminations of the fifth nerve in the jaw cause reflex eye symptoms.

Many of the inflammatory diseases of the eye are either the result or the manifestation of general bodily disease. That a localized focus of pyogenic organisms growing and confined in some distant organ is a frequent cause of inflammation in the eye is, I believe, open to question. That it may occasionally be the cause of such inflammation in the eye is doubtless true; that the finding of such a focus of infection in conjunction with an inflamed eye proves the focus of infection to be the cause of the inflamed eye is not true. It is not true even tho no other cause for the inflammation is found. It is a conclusion based upon exclusion. To be absolutely accurate I may say that it is a conclusion based upon possibly partial exclusion. It is a conclusion upon which both you and I often base our treatment. But it is a conclusion after which a question mark must always be placed.

The beauty of the theory lies not so much in its scientific accuracy as in the fact that it calls our attention and the attention of our patient to a condition which never does good and can only do harm; which tends toward evil and should be eradicated. It should be eradicated whether or not it is the cause of the pathological manifestation for which we are seeking a cause.

These brief remarks are not intended to decry the whole theory of focal infection but to check its unbridled acceptance as the final etiology in all disease. 